\title{
Erratum
}

\section{Directional weighted spatial fuzzy C-means for segmentation of brain MRI images}

\author{
(Journal of X-ray Science \& Technology, \\ Vol 27, No 6, pp. 1087-1099, 2019)
}

Sajid Ullah Khan ${ }^{\mathrm{a}, *}$, Imran Ullah $^{\mathrm{b}}$, Imran Ahmed ${ }^{\mathrm{c}}$, Ali Imran ${ }^{\mathrm{d}}$ and Najeeb Ullah ${ }^{\mathrm{e}}$

${ }^{\mathrm{a}}$ The University of Lakki Marwat, KPK, Pakistan

${ }^{\mathrm{b}}$ Harbin Engineering University, China

${ }^{\mathrm{c}}$ Institute of Management Sciences, Peshawar, Pakistan

${ }^{\mathrm{d}}$ University of Science and Technology, Bannu, Pakistan

${ }^{\mathrm{e}}$ CECOS University of IT and Emerging Sciences, Peshawar, Pakistan

The authors identified errors in this recently published paper [1] and would like to make following two changes.

1. Original title of this paper does not accurately reflect this work. A new title should be "A spatial fuzzy C-means algorithm for segmentation of brain MRI images."

2. There are errors in the reported segmentation accuracy data of the proposed framework with brain in Table 2. The corrected Table 2 is shown in the following.

Table 2

Results comparison with existing methods over 51 simulated T1-weighted images using Segmentation Accuracy (SA) measure

\begin{tabular}{llcccc}
\hline S. No & Methods & \multicolumn{2}{c}{ Avg. Accuracy of Tissues Segmentation \% } & \multicolumn{2}{c}{ Average (SA) } \\
\cline { 3 - 5 } & & GM & WM & CSF & Complete \\
\hline 1 & sFCM [24] & 87.20 & $89 \%$ & $83.10 \%$ & $86.43 \%$ \\
2 & FGFCM [25] & 78.30 & 89.40 & 77.90 & $81.87 \%$ \\
3 & ASIFC [26] & 88.50 & 92.60 & 85.60 & $88.90 \%$ \\
4 & csFCM [27] & 89.00 & 94.70 & 88.40 & $90.70 \%$ \\
5 & Proposed framework with brain & 95.21 & 95.12 & 92.15 & $92.14 \%$ \\
\hline
\end{tabular}

\section{Reference}

[1] S.U. Khan, I. Ullah, I. Ahmed, A. Imran and N. Ullah, Directional weighted spatial fuzzy C-means for segmentation of brain MRI images, J Xray Sci Technol 27 (2019), 1087-1099.

\footnotetext{
${ }^{*}$ Corresponding author: Sajid Ullah Khan, CECOS University of IT and Emerging Sciences, Peshawar 28300, Pakistan. Tel.: +92 3339323054; E-mail: sajdi786@yahoo.com.
} 